\title{
Evaluation of Skin Elasticising Topical Formulations by a Torsiometric Test
}

\author{
Adele Sparavigna1, Francesco Scarci², Federico Mailland ${ }^{2}$ \\ ${ }^{1}$ Derming, Clinical Research and Bioengineering Institute, Monza, Italy \\ ${ }^{2}$ Scientific Department, Polichem S.A., Lugano, Switzerland \\ Email: fscarci@polichem.com
}

Received 28 January 2014; revised 25 February 2014; accepted 5 March 2014

Copyright (C) 2014 by authors and Scientific Research Publishing Inc.

This work is licensed under the Creative Commons Attribution International License (CC BY).

http://creativecommons.org/licenses/by/4.0/

(c) (i) Open Access

\begin{abstract}
Background/Purpose: In the frame of a development of a formulation to be used in perineal massage in pregnant women, a screening program was built up among compositions with elasticising properties. Methods: This was an open-label, controlled study in 21 volunteers. The participants applied once, on the volar surface of both forearms, five formulations with elasticising properties (test products 1 to 5) and a reference standard (test product 6 ). The formulations were applied on three sites on each forearm that were randomly assigned. Skin elasticising parameters were evaluated using the Dermal Torque Meter, at baseline and 30 minutes after application. Results: Among the test products, only test product 2 significantly increased skin elasticity compared with the baseline value, with a trend toward superiority versus the reference standard $(+24 \%$ vs. $+13 \%$, respectively). There was a significant increase in elastic recovery of $23 \%$ with test product 2 , compared with a significant increase of $13 \%$ with the reference standard. Conclusion: Test product 2, comprising a thiolated compound and a mixture of esters of fatty acids, had the greatest elasticising effect. Compared with the other tested formulations, product 2 led to a greater increase in extensibility and faster elastic recovery of the skin on the volar surface of the forearm.
\end{abstract}

\section{Keywords}

Emollient; Lubricant and Elasticising Properties; Skin Elasticity; Prevention of Lacerations

\section{Introduction}

The sponsor developed a composition specifically directed to improve tissue elasticity, in order to prevent lacerations during delivery. The first step concerned the selection of a non-pharmaceutical product able to improve the elasticity of the skin. 
In order to investigate the biomechanical and elasticizing properties of the composition, it set up a screening program aiming to find a new medical device acting through a physical mechanism.

The lubricating and moisturizing properties of paraffin are well-known [1], as those of fats, including the esters of glycerol and those of animal or vegetable steroids [2]. In the field of such discovery, a number of compositions were prepared, containing thiolated compounds and/or a mixture of esters of fatty acids, antioxidants and glycerol, with the aim to find a "lead" with maximum elasticising, soothing and lubricating properties. Thiols have been chosen for their capability, by reducing the S-S bonds, to modify the quaternary composition of proteins [3] and to allow their extension, therefore preventing stretching traumas.

\section{Materials and Methods}

The study was an open-label, controlled, pharmaco dynamic investigation of five topical test formulations compared with a reference standard. According to the study protocol, at least 20 healthy female volunteers, aged between 18 and 55 years, had to be recruited.

The protocol was approved by the ethics committee (Comitato Etico Indipendente Multidisciplinare Derming) and conducted according to ICH-GCP guidelines and to the Declaration of Helsinki. Written informed consent was obtained by all volunteers before starting any study procedure. The most important exclusion criteria were pregnancy, breastfeeding, presence of concomitant dermatological conditions and the use of any topical treatment in the test skin area. Each volunteer had to apply once, on the volar surface of the forearm, all six formulations tested in the study. The volar surface of each forearm was divided into three sites: proximal, middle and distal. The six sites on which the formulations were tested were randomly assigned, according to a randomisation list generated by a computerised system. In order to increase absorption, the formulations were applied and gently massaged in order to favour the absorption.

The general compositions of the test products were as follows:

-Test product 1: thiolated compound (carboxymethyl cysteine), antioxidants, moisturizer and thickener;

-Test product 2 (Elastolabo ${ }^{\circledR}$ [Polichem SA-Lugano-Switzerland]): thiolated compound (carboxymethyl cysteine), antioxidants, paraffin and a mixture of organic fatty acid esters;

-Test product 3: a hydrogel consisting of organic fatty acid esters, moisturiser and antioxidants;

-Test product 4: an oil-in-water emulsion;

-Test product 5: a water-in-oil emulsion;

-Reference standard: $100 \%$ white soft paraffin.

A gel of white soft paraffin was chosen as the reference standard, as it is considered to be the gold standard emollient [1]. The skin elasticising property was evaluated at baseline and 30 minutes after the application of the test product or the reference standard, using the Dermal Torque Meter (Dia-Stron Ltd., UK) (Figure 1).

The working principle of the torque is based on the torsion achieved in vivo on a specific skin section, by a mechanical probe, made by two concentric rings adhered to the skin by a piece of double-sided adhesive tape of

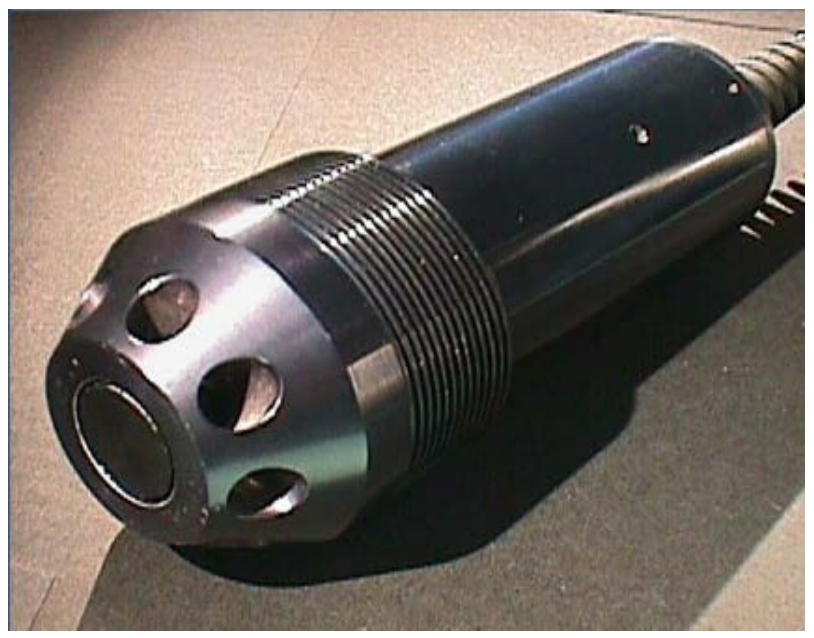

Figure 1. Dermal torque meter. 
the same shape. A $1 \mathrm{~mm}$ gap was kept between the central disk and the concentric stationary ring to allow movement. Rotation of the inner disk relative to the outer ring achieved continuous torsion of the skin. The rotational motion was interrupted as soon as the opposite force from the skin balanced the initial torsion applied, corresponding to $9 \mathrm{mN} \cdot \mathrm{m}$. The mechanical properties of the skin were determined by application of torque to the central disk for $1 \mathrm{~s}$ and carefully measuring the degree of rotation during torsion (torque on, $\tau_{\mathrm{on}}$ ) and the degree of back rotation at torsion suspension (torque off, $\tau_{\text {off }}$ ). The torsional measurement was defined by the torsion angle $\theta$ at the time of maximum extensibility and at the time of elastic recovery.

The plasto-elasticity of the skin (shown in Figure 2) was calculated as follows: skin elasticity (Ur/Uf) was defined as the ratio between immediate elastic recovery and maximum extensibility, where $\mathrm{Ue}=$ immediate extensibility (torque on at $0.02 \mathrm{~s}$ ); $\mathrm{Uf}=$ maximum extensibility (torque on at $0.9 \mathrm{~s}$ ); $\mathrm{Ur}=$ immediate elastic recovery (torque off at $0.2 \mathrm{~s}$ ); and $\mathrm{Uv}$ = viscoelasticity; elastic recovery $(\mathrm{Ur} / \mathrm{Ue})$ was defined as the ratio between immediate recovery and immediate extensibility.

\section{Results}

Twenty-one healthy female volunteers, aged 24 to 55 years (mean \pm SD, $45.10 \pm 9.82$ ), were enrolled in the study, after providing written informed consent. The percentage change in torsiometric parameters relative to baseline at 30 min after application of the test products on the volar surface of the forearm is summarised in Figure 3.

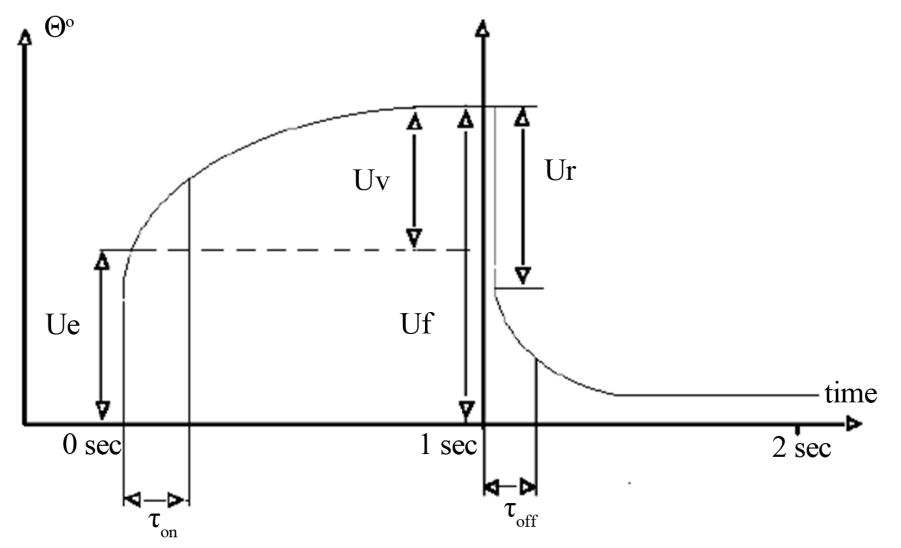

Figure 2. Graphic representation ofthe plasto-elasticity parameters.

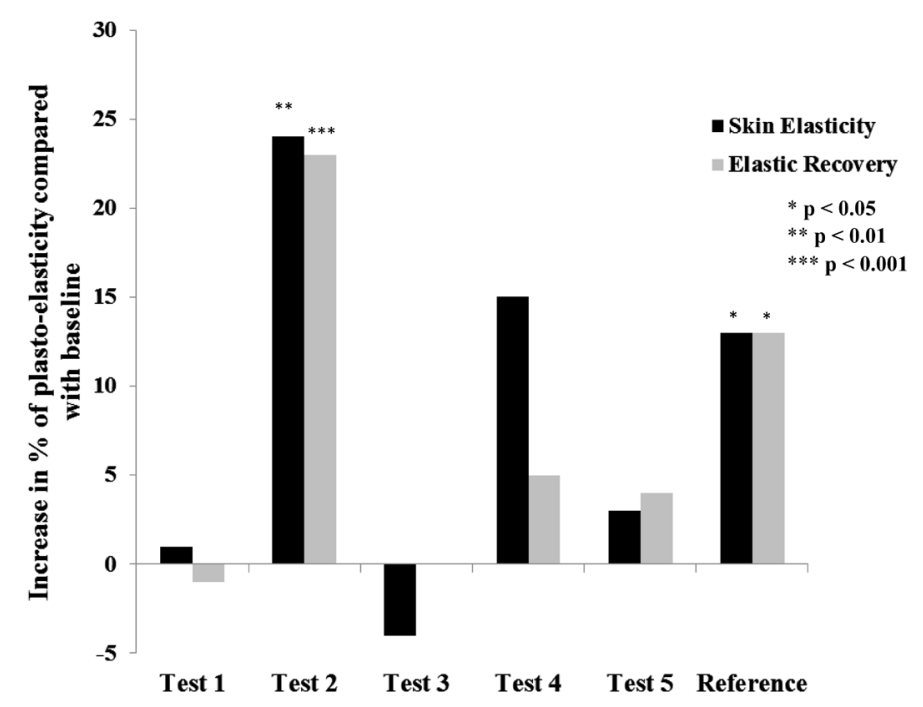

Figure 3. Change vs. Baseline of skin elasticity and elastic recovery in all tested products. 
Skin elasticity was not affected by test product 1 , slightly but not significantly reduced by test product 3 , and was slightly but not significantly increased by test products 4 and 5 . Among the test products, only test product 2 significantly increased skin elasticity compared with the baseline value (24\% increase in Ur/Uf; mean \pm SD, $0.319 \pm 0.060$ and $0.396 \pm 0.124$ at baseline and 30 minutes after application, respectively; $\mathrm{p}<0.01$ Student's $\mathrm{t}$ test for paired data). The reference standard significantly increased skin elasticity, but the increase $(+13 \% \mathrm{Ur} / \mathrm{Uf}$; $0.366 \pm 0.075$ and $0.414 \pm 0.089$ at baseline and 30 minutes after application, respectively; $\mathrm{p}<0.05$ ) was smaller compared with test product 2 .

Elastic recovery was not affected by test product 1 or test product 3, and was slightly but not significantly increased by test products 4 and 5 . A significant increase of $23 \%$ was recorded for test product 2 , with mean \pm SD $\mathrm{Ur} / \mathrm{Ue} 0.596 \pm 0.097$ and $0.735 \pm 0.147$ at baseline and 30 minutes after application, respectively $(\mathrm{p}<0.001$ Student's t test). The reference standard led to a significant increase of $13 \%$ in Ur/Ue ( $0.664 \pm 0.095$ and $0.752 \pm$ 0.102 at baseline and 30 minutes after application, respectively; $\mathrm{p}<0.05$ Student's $\mathrm{t}$ test).

The statistical analysis of all parameters was performed only for the two formulations that showed a significance difference between the end and baseline values for skin elasticity and for elastic recovery, namely test product 2 and the reference standard (Table 1 and Figure 4).

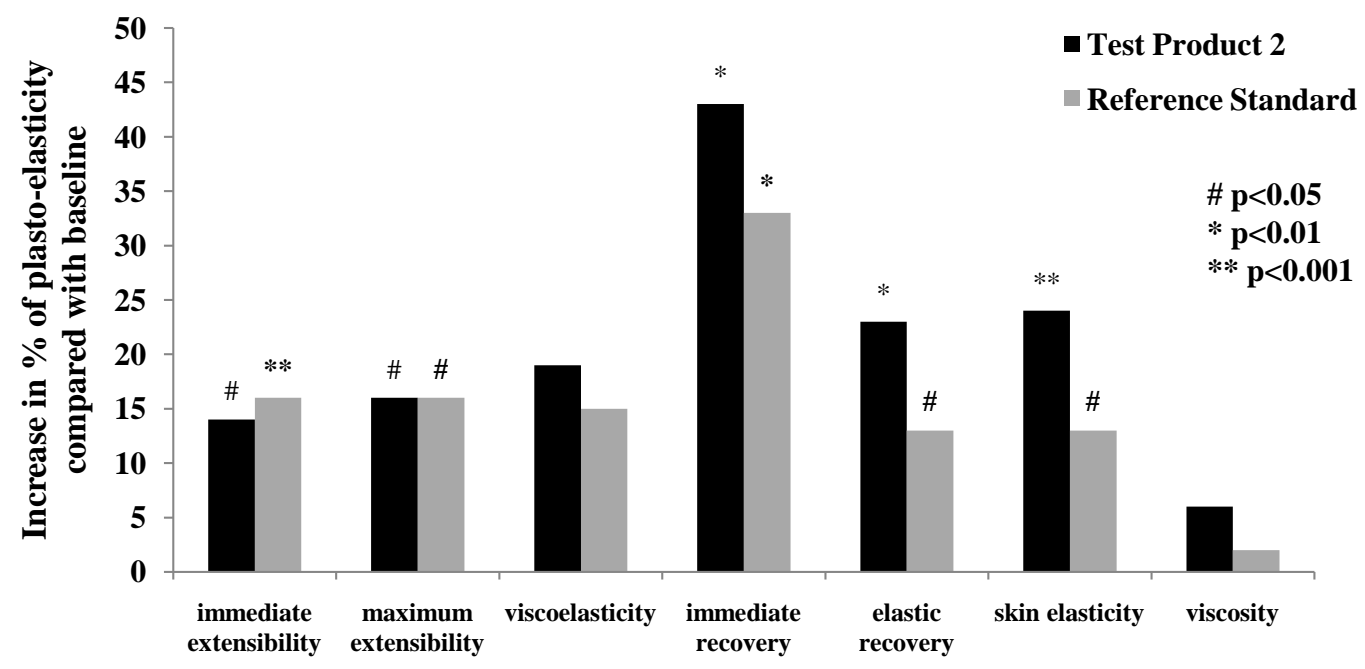

Figure 4. Change vs. baseline of all skin plasto-elasticity parameters for test product 2 vs. Reference standard.

Table 1. Parameters of skin plasto-elasticty measured by torquemeter before and 30 minutes after the application of test product 2 and reference standard. Data are means \pm SD.

\begin{tabular}{|c|c|c|c|c|c|c|c|}
\hline & \multirow[b]{2}{*}{ Parameter } & \multicolumn{3}{|c|}{ Test Product 2} & \multicolumn{3}{|c|}{ Standard Reference } \\
\hline & & Baseline & $\begin{array}{c}\text { After } \\
\text { Treatment }\end{array}$ & $\begin{array}{c}\text { P between } \\
\text { times }\end{array}$ & Baseline & $\begin{array}{c}\text { After } \\
\text { Treatment }\end{array}$ & $\begin{array}{c}\text { P between } \\
\text { times }\end{array}$ \\
\hline & Ue-Immediate Extensibility & $1.426 \pm 0.350$ & $1.624 \pm 0.409$ & $<0.05$ & $1.614 \pm 0.491$ & $1.869 \pm 0.577$ & $<0.01$ \\
\hline \multirow[t]{2}{*}{ Torque ON } & Uf-Maximum Extensibility & $2.664 \pm 0.624$ & $3.102 \pm 0.836$ & $<0.05$ & $2.954 \pm 0.918$ & $3.416 \pm 0.940$ & $<0.05$ \\
\hline & Uv-Viscoelasticity & $1.238 \pm 0.294$ & $1.479 \pm 0.582$ & n.s. & $1.340 \pm 0.492$ & $1.547 \pm 0.547$ & n.s. \\
\hline Torque OFF & Ur-Immediate Recovery & $0.842 \pm 0.236$ & $1.204 \pm 0.427$ & $<0.001$ & $1.064 \pm 0.358$ & $1.413 \pm 0.507$ & $<0.001$ \\
\hline Ur/Ue & Elastic Recovery & $0.596 \pm 0.097$ & $0.735 \pm 0.147$ & $<0.001$ & $0.664 \pm 0.095$ & $0.752 \pm 0.102$ & $<0.05$ \\
\hline $\mathbf{U r} / \mathbf{U f}$ & Skin Elasticity & $0.319 \pm 0.060$ & $0.396 \pm 0.124$ & $<0.01$ & $0.366 \pm 0.075$ & $0.414 \pm 0.089$ & $<0.05$ \\
\hline \multirow[t]{3}{*}{ Uv/Ue } & Viscosity & $0.876 \pm 0.116$ & $0.928 \pm 0.298$ & n.s. & $0.837 \pm 0.192$ & $0.855 \pm 0.252$ & n.s. \\
\hline & $\tau_{\mathrm{on}}$ & $0.044 \pm 0.010$ & $0.051 \pm 0.022$ & n.s. & $0.042 \pm 0.016$ & $0.045 \pm 0.020$ & n.s. \\
\hline & $\tau_{\text {off }}$ & $0.043 \pm 0.010$ & $0.028 \pm 0.009$ & $<0.001$ & $0.035 \pm 0.010$ & $0.025 \pm 0.005$ & $<0.001$ \\
\hline
\end{tabular}


The application of test product 2 or the reference standard increased significantly, relative to baseline, the parameters of immediate extensibility, maximum extensibility, skin elasticity, elastic recovery and immediate recovery. However, they did not significantly affect either viscosity or viscoelasticity. The parameters of extensibility (immediate extensibility and maximum extensibility) and $\tau_{\text {on }}$ were increased by a similar amount by both formulations. On the contrary, test product 2 showed a trend toward superiority compared with the reference standard in increasing skin elasticity ( $+24 \%$ vs. $+13 \%$, respectively), elastic recovery $(+23 \%$ vs. $+13 \%$, respectively), immediate recovery ( $+43 \%$ vs. $+33 \%$, respectively), and in reducing $\tau_{\text {off }}(-35 \%$ vs. $-29 \%$, respectively).

\section{Discussion and Conclusion}

Our results show the elasticising properties of test product 2 in terms of increased extensibility and faster elastic recovery, on the volar forearm surface after application. The formulation of test product 2 was designed to contain as the main ingredients: 1) a thiolated compound, to break disulphide bonds and allow the extension of keratin; 2) a mixture of esters of fatty acids, to take advantage of the emollient and lubricating properties of fats.

The screening was performed by evaluating the elasticising effects of different formulations with emollient properties, as it is well known that emollients have a significant effect on skin elasticity [4]. Furthermore, formulations containing a thiolated compound were investigated, because of its ability to increase the extensibility of proteins that are rich in cysteine and disulphide bonds, including keratin. The screening demonstrated that neither the emollients nor the thiolated compound alone had skin elasticising properties similar to those of the reference standard, $100 \%$ soft white paraffin; on the contrary, the combination of emollients and thiolated compound had a synergic effect on elasticising tissues, with a trend toward superiority versus the reference standard. The results of this screening have met the objective to find a lead with enhanced elasticising properties on superficial tissues compared with the reference standard. This product, which is devoid of pharmacological activity, could be used during pregnancy without risks for the foetus. As a matter of fact, it has been used in a clinical trial in pregnant women, confirming the ability to prevent rate and severity of lacerations during delivery. Interim data have been recently published [5].

\section{Acknowledgements}

The Company Sinerga (Pero, Italy) is gratefully acknowledged for having provided the screened formulations.

\section{Conflict of Interest}

The clinical study was $100 \%$ supported by Polichem SA that is the holder of all rights related to the study. F.S. and F.M. are employees of Polichem SA.

\section{References}

[1] Morrison, D.S. (1996) Petrolatum: A Useful Classic. Cosmetics and Toiletries Magazine, 111, 59-69.

[2] Uchimoto, T., et al. (2010) Lubrication Properties of Potential Alternative Lubricants, Glycerin Fatty Acid Esters, to Magnesium Stearate. International Journal of Pharmaceutics, 386, 91-98. http://dx.doi.org/10.1016/j.ijpharm.2009.11.001

[3] Aćimović, J.M., Stanimirović, B.D. and Mandić, L.M. (2009) The Role of the Thiol Group in Protein Modification with Methylglyoxal. Journal of the Serbian Chemical Society, 74, 867-883. http://dx.doi.org/10.2298/JSC0909867A

[4] Proksch, E. and Lachapelle, J.M. (2005) The Management of Dry Skin with Topical Emollients-Recent Perspectives. Journal der Deutschen Dermatologischen Gesellschaft, 3, 768-774. http://dx.doi.org/10.1111/j.1610-0387.2005.05068.x

[5] Reggiardo, G., Fasani, R. and Mignini, F. (2012) Multicentre, Open Label Study to Evaluate the Efficacy and Tolerability of a Gel (Elastolabo ${ }^{\circledR}$ ) for the Reduction of the Incidence of Perineal Traumas during Labour and Related Complications in the Postpartum Period. Trends in Medicine, 12, 143-149. 